\title{
Correlation between uniocular deviation and duction changes following different decompression surgeries in thyroid eye disease
}

Jie Guo ${ }^{1,2,3 \dagger}$, Xiaofeng $\mathrm{Li}^{1,2,3+}$, Ruiqi Ma ${ }^{1,2,3}$ and Jiang Qian ${ }^{1,2,3^{*}}$

\begin{abstract}
Background: Postoperative ocular imbalance is an important problem for orbital decompression surgery in thyroid eye disease (TED). The aim of this study was to evaluate the changes in unilateral ocular deviation and duction following orbital decompression and discuss the biomechanics of ocular imbalance.

Methods: Fifty-four TED patients who underwent unilateral orbital decompression were included. Fifteen patients underwent 1-wall (deep lateral wall) decompression, 18 patients underwent 2-wall (deep lateral and medial wall) decompression and 21 patients underwent 3-wall (deep lateral, medial and inferior wall) decompression. Objective and subjective deviation of the operated eyes were evaluated using the prism test and synoptophore, respectively. Ocular ductions were measured using Hirschberg's method. The diameters of the extraocular rectus were measured by computed tomography.
\end{abstract}

Results: Ocular deviation and duction showed no significant difference after 1-wall decompression $(p=0.25-0.89)$. Esotropia increased after 2-wall decompression ( $p=0.001-0.02$ ), and hypotropia increased after 3-wall decompression $(p=0.02$ ). Adduction increased but abduction decreased following 2 -wall and 3-wall decompression $(p<0.05)$. Infraduction increased following 3-wall decompression $(p<0.001)$. Additionally, the increase in esotropia was significantly correlated with the increase in adduction and with the decrease in abduction $(r=0.37-0.63, p<$ 0.05). There were significant correlations between the diameter of the medial rectus and the increase in esotropia, the increase in adduction and the decrease in abduction postoperatively $(r=0.35-0.48, p<0.05)$.

Conclusions: The changes in ocular deviation and duction were different after 1-wall, 2-wall and 3-wall orbital decompression. The increased contractile force of the rectus may be an important reason for strabismus changes after orbital decompression surgery.

Keywords: Thyroid eye disease, Orbital decompression, Ocular duction, Strabismus

\footnotetext{
* Correspondence: qianjiang58@163.com

${ }^{\dagger}$ Jie Guo and Xiaofeng Li contributed equally to this work.

${ }^{1}$ Eye Institute and Department of Ophthalmology, Eye \& ENT Hospital, Fudan University, Shanghai, China

${ }^{2} \mathrm{NHC}$ Key Laboratory of Myopia (Fudan University, Key Laboratory of Myopia,

Chinese Academy of Medical Sciences, No. 83 Fenyang Road, Shanghai 200031, China

Full list of author information is available at the end of the article
}

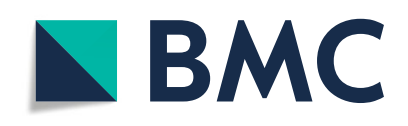

(- The Author(s). 2021 Open Access This article is licensed under a Creative Commons Attribution 4.0 International License, which permits use, sharing, adaptation, distribution and reproduction in any medium or format, as long as you give appropriate credit to the original author(s) and the source, provide a link to the Creative Commons licence, and indicate if changes were made. The images or other third party material in this article are included in the article's Creative Commons licence, unless indicated otherwise in a credit line to the material. If material is not included in the article's Creative Commons licence and your intended use is not permitted by statutory regulation or exceeds the permitted use, you will need to obtain permission directly from the copyright holder. To view a copy of this licence, visit http://creativecommons.org/licenses/by/4.0/. The Creative Commons Public Domain Dedication waiver (http://creativecommons.org/publicdomain/zero/1.0/) applies to the data made available in this article, unless otherwise stated in a credit line to the data. 
Orbital decompression surgery is a crucial treatment for thyroid eye disease (TED). It is effective for treating proptosis, exposure keratopathy and optic neuropathy. Various surgical techniques have been utilized, including medial, inferior, lateral orbital wall removal with or without intraconal fat removal.

Currently, orbital decompression is more focused on the risks of ocular imbalance after surgery. New-onset diplopia (NOD) and worsening of the pre-existing strabismus are the major problems that have been widely studied. Inferomedial decompression is associated with a relatively high risk of consecutive diplopia and globe displacement (ranging from 9.5 to $73 \%$ ), whereas balanced decompression (ranging from 10 to $45 \%$ ) and deep lateral decompression (ranging from 2.6 to $8 \%$ ) are considered to lessen the imbalanced shifting of extraocular muscles (EOM) and to reduce the likelihood of NOD [1-4]. However, studies on the exact changes in ocular deviation and duction after unilateral orbital decompression are lacking. Studying the relationship between ocular duction and deviation may help to understand the biomechanics of ocular imbalance [5, 6].

This study analysed the changes in uniocular deviation and duction after 1-wall, 2-wall and 3-wall decompression surgery, and assessed the correlations between them.

\section{Patients and methods}

The study was conducted at Eye and ENT Hospital of Fudan University between March 2016 and May 2018. The TED patients who underwent orbital decompression surgery, including 1-wall (deep lateral wall), 2-wall (deep lateral and medial wall) and 3-wall (deep lateral, medial and inferior wall) decompression were enrolled. The indications for orbital decompression included disfiguring proptosis, exposure keratopathy and compressive optic neuropathy.

To evaluate the exact changes in ocular deviation and duction following decompression surgeries, only the patients who underwent unilateral surgery were collected, including the patients who had unilateral proptosis, the patients who had asymmetric proptosis and intended to undergo decompression surgeries at different times, and the patients who had unilateral surgery 6 months previously and needed a decompression surgery for the other eye.

The exclusion criteria included a history of orbital surgery of the studied eyes, low vision with an inability to complete the strabismus examinations, and bilateral orbital decompression performed within a short time. The strabismus and motility examinations were performed within 1 week before the surgery and were repeated several months after the surgery. The following data were obtained: gender, age, disease duration, bestcorrected visual acuity (logMAR), clinical activity score
(CAS), Hertel exophthalmometry, history of thyroid disease, and iodine 131 and corticosteroid treatment.

The study adhered to the tenets of the Declaration of Helsinki and was approved by the Ethics Committee of Fudan University. Informed consent was obtained from all patients in the study.

\section{Surgical technique}

All decompression surgeries were performed by a single surgeon. Deep lateral wall decompression was performed through an eyelid crease incision. The lacrimal gland fossa and the greater wing of the sphenoid bone were removed using a high-speed drill. The outer region of the zygomatic bone lateral to the inferior orbital fissure (basin region) was additionally removed in some patients. The periorbita was incised to allow orbital fat to herniate into the surrounding spaces. Medial wall decompression was performed through a transcaruncular incision. Medial and posterior ethmoidectomy was performed and the periorbita was incised. For inferior wall decompression, a small region of the posteromedial orbital floor was removed. The ethmoid-maxillary bony strut was preserved. The extent of osteotomy was individualized based on the degree of proptosis. For some patients, the inferolateral intraconal fat was removed with volumes ranging from 0.5 to $1.5 \mathrm{ml}$.

\section{Ocular deviation and duction examinations}

Strabismus examinations were performed by an experienced orthoptist. Objective deviation was measured with the prism test, and subjective deviation was measured using a synoptophore. Horizontal deviation was recorded as a positive value when the operated eye exhibited esotropia compared with the fellow eye and was recorded as a negative value when exotropia was present. Vertical deviation was recorded as a positive value when the operated eye exhibited hypertropia compared with the fellow eye and was recorded as a negative value when hypotropia was present. Torsional deviation was recorded as a positive value when the operated eye exhibited excyclotropia and was recorded as a negative value when incyclotropia was present.

Ocular duction was evaluated using Hirschberg's method [7]. The clinician shined a pen light in line with the eye being examined. The patient was asked to look in four cardinal directions as far as possible, and the position of the light was viewed on the surface of the eye: at limbus $=45^{\circ}$, between limbus and pupil $=30^{\circ}$, and at pupil edge $=15^{\circ}$. The clinician estimated $5^{\circ}$ intervals by dividing the space into thirds between these designations, and the nearest $5^{\circ}$ was recorded (Fig. 1).

\section{Extraocular muscle evaluations}

Computed tomography (CT) scans with $0.75 \mathrm{~mm}$ slice thickness were performed within 1 month before surgery. 


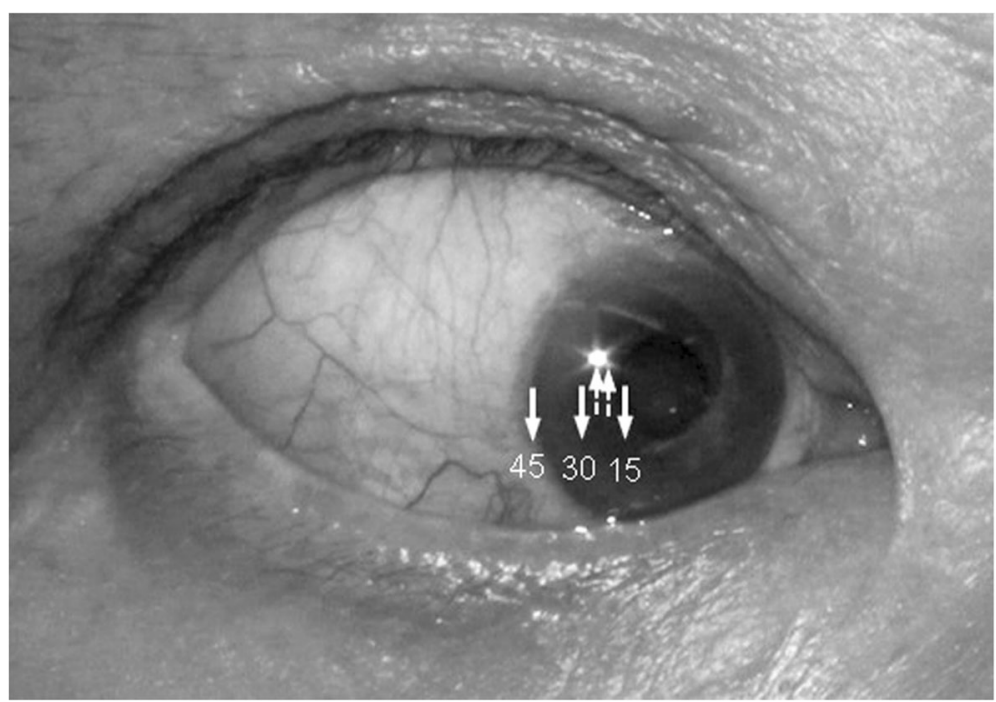

Fig. 1 Ocular duction was evaluated using Hirschberg's method. Light target on the surface of the eye at limbus $=45^{\circ}$, between limbus and pupil $=30^{\circ}$, and at pupil edge $=15^{\circ}$ (solid arrow). The space between these designations was divided into thirds, and intermediate duction was estimated to the nearest $5^{\circ}$ (dotted arrow). This patient is looking inward at $25^{\circ}$

The maximum diameters of the medial rectus (MR) and lateral rectus (LR) were measured from axial CT images. The maximum diameters of the inferior rectus and superior muscle groups, including the superior rectus and levator palpebrae superioris, were measured from coronal CT images.

\section{Statistical analysis}

The mean values are presented with standard deviations. The paired $t$ test was used to compare the preoperative and postoperative measurements. ANOVA was used to compare the preoperative measurements between different groups. Pearson correlation analysis was used to analyse the correlations between ocular deviations, ductions and rectus diameters. The chi-square test was used to compare the decompression extent between the 2 -wall and 3-wall groups. All differences with a value of $p<0.05$ were considered statistically significant. The analyses were performed using SPSS.

\section{Results}

Fifty-four patients (26 men and 28 women) were included in the study. The mean age was $51.7 \pm 12.5$ years (18-72 years), and the mean CAS was $1.6 \pm 1.1$ (0-3). Fifteen patients $(27.8 \%)$ underwent 1 -wall decompression surgery, 18 patients (33.3\%) underwent 2wall decompression surgery and 21 patients (38.9\%) underwent 3-wall decompression surgery. The mean follow-up duration was $4.2 \pm 0.9$ months (from 4 to 9 months). The basin region was removed more frequently in the 3-wall decompression group than in the 2 -wall decompression group $(p=0.003)$.
The mean proptosis reductions were $2.5 \pm 0.5 \mathrm{~mm}$, $3.2 \pm 1.1 \mathrm{~mm}$, and $4.4 \pm 1.1 \mathrm{~mm}$ after 1-wall, 2-wall and 3-wall orbital decompression, respectively. The mean visual acuity improved after 2-wall decompression surgery (logMAR $0.37 \pm 0.4$ preoperatively and $\log$ MAR $0.14 \pm 0.14$ postoperatively, $p=0.04$ ) and 3-wall decompression surgery (logMAR $0.41 \pm 0.41$ preoperatively and $\log$ MAR $0.22 \pm 0.25$ postoperatively, $p=0.001$ ). The mean visual acuity did not significantly change after 1 -wall decompression surgery (logMAR $0.04 \pm 0.06$ preoperatively and $\log$ MAR $0.03 \pm 0.05$ postoperatively, $p=0.336$ ).

No significant difference was found in preoperative ocular deviation between the three groups $(p>0.05)$. There was a significant difference in preoperative adduction between the three groups $(p<0.05)$. The patients who underwent 2-wall and 3-wall decompression had more limitations in preoperative abduction than those who underwent 1 -wall decompression $(p<0.05)$. The patients who underwent 3-wall decompression had more limitations in preoperative infraduction and supraduction than those who underwent 1-wall decompression $(p<0.05)$.

The changes in objective and subjective strabismus following different orbital decompression are listed in Table 1. Horizontal and vertical deviation showed no significant difference after 1-wall decompression $(p=0.25$ 0.89 ). Most of the patients who underwent 1-wall decompression had a minor change in horizontal deviation, except for two patients who had enlarged MR preoperatively. Esotropia increased significantly in the patients who underwent 2-wall decompression $(p=0.001-0.02)$, and hypotropia increased in most of the patients who 


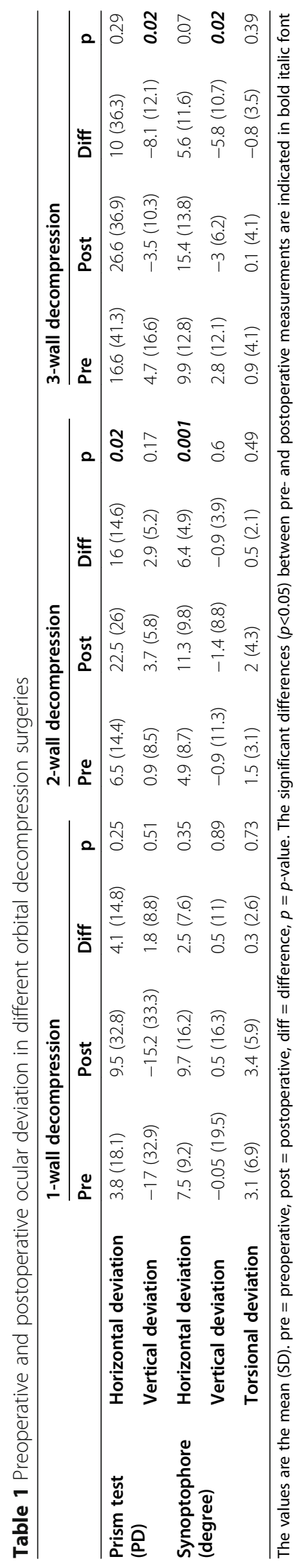


underwent 3-wall decompression $(p=0.02)$. Esotropia had an increasing tendency after 3-wall decompression but the difference did not reach statistical significance. Torsional deviation showed no significant difference postoperatively in any of the groups $(p=0.39-0.73)$.

The changes in ocular ductions after different orbital decompression are shown in Table 2 and Fig. 2. There was no significant change in ocular motility after 1-wall decompression $(p=0.083-0.667)$. Adduction significantly increased after 2-wall and 3-wall decompression $(p<0.05)$, whereas abduction significantly decreased $(p<0.05)$. Infraduction significantly increased after 3 wall decompression $(p<0.001)$.

For all the patients who underwent orbital decompression, the increase in postoperative esotropia had a significant correlation with the increase in adduction (prism test: $r=0.48, p<0.001$, synoptophore: $r=0.63$, $p<0.001$ ) and with the decrease in abduction (prism test: $r=0.37, p=0.008$, synoptophore: $r=0.49, p<0.001$ ) (Fig. 3). Additionally, there were significant correlations between the diameter of MR and the increase in esotropia (prism test: $r=0.47, p<0.001$, synoptophore: $r=0.48, p<$ $0.001)$, the increase in adduction $(r=0.4, p=0.004)$ and the decrease in abduction $(r=0.35, p=0.011)$. No significant difference was found between the changes in vertical deviations and ocular ductions $(p>0.1)$.

In some subjects who had an obviously increased esotropia postoperatively, the MR tended to become thicker after surgery, whereas its position exhibited no obvious change (Fig. 4).

\section{Discussion}

Previous reports on strabismus changes following orbital decompression primarily investigated patients who underwent bilateral surgeries. Our study provided an evaluation of the postoperative changes in unilateral ocular deviation and duction, which may aid in the understanding of the biomechanics of ocular imbalance.

Simon et al. reported that the number of patients with esotropia increased after deep lateral decompression [8], and new-onset esotropia was reported to range from 10 to $18 \mathrm{PD}$ [9]. Goldberg et al. suggested that esotropia could worsen after lateral decompression due to LR weakness [10]. However, in another of their studies, the patients exhibited minor exotropia after deep lateral decompression with an average value of 3.7 PD [3]. Additionally, Shani Golan et al. found that the changes in strabismus varied after inferolateral decompression [11]. These discrepancies resulted from different disease severities, preoperative strabismus and surgical techniques. In our study, most of the patients who underwent 1-wall decompression had only a minor change in horizontal deviation except for two patients who had enlarged MR.
New-onset esotropia has been reported to occur after balanced decompression, with the incidence ranging from 4 to 20 PD [9]. In our study, esotropia increased in nearly all patients who underwent 2-wall decompression. Esotropia had an increasing tendency after 3-wall decompression but the difference did not reach statistical significance. The volume of bone removed from the lateral wall was expanded in most patients who underwent 3 -wall decompression, including the basin region [10]. We supposed that the expansion of lateral wall decompression could further compensate for the imbalanced shift in orbital tissue and reduce the potential esotropia. Additionally, the preoperative adduction was more restricted in the patients who underwent 3-wall decompression, and the increased stiffness and contractile force of LR could be another reason for the reduction in the postoperative esotropia. A small but significant increase in hypotropia was observed in nearly all patients who underwent 3-wall decompression. Although the bone removed from the orbital floor was limited to the most posteromedial portion adjacent to the ethmoid bone, and the strut was preserved in our study [12].

Although slight differences existed between the results of the synoptophore and prism test due to binocular fusion, accommodation and the kappa angle, the synoptophore test may be better related to the subjective symptom of diplopia than the prism test and could be considered an important component of strabismus evaluation in TED. The change in torsional deviation following orbital decompression has not been discussed previously. Our study showed that torsional deviation did not significantly change after orbital decompression.

The development of oculomotor imbalance following orbital decompression is a multifactorial problem. Michael et al. reported a significant decrease in abduction after 3-wall decompression [13]. Inna et al. reported that adduction and abduction decreased after coronal 3-wall decompression, whereas infraduction decreased after swinging eyelid 3-wall decompression, possibly due to the difference in decompression extent [14]. Rootman et al. reported that abduction worsened after orbital decompression [15]. In our study adduction increased but abduction decreased after 2-wall and 3-wall decompression. The infraduction increased after 3-wall decompression.

It has been proven that the force and elasticity of the extraocular rectus change in TED patients, as well as that the limitation of abduction is not only due to the fibrosis of MR but also to the increased contractile force of MR [16, 17]. In previous studies, the centrifugal displacement of the rectus path was believed to be the main reason for the motility disturbance after decompression [13], however, we found that the MR became thicker without displacement in some patients who had an obvious esotropia postoperatively, which may indicate 


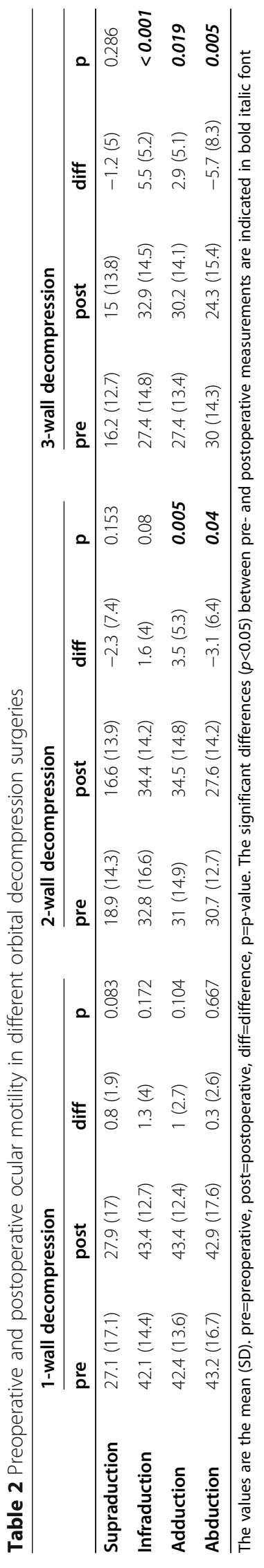




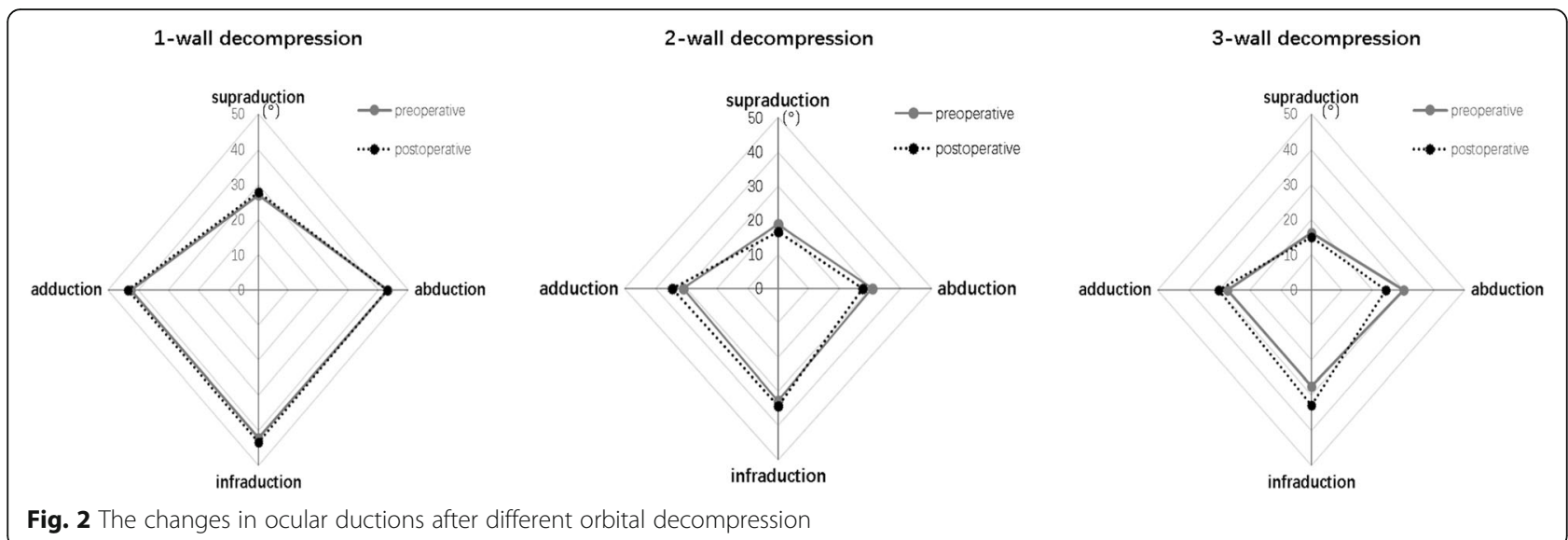

the increased contractile force of the MR pulling the globe after surgery. That may be why the increase in esotropia correlated with the increase in adduction and the decrease of abduction in the study. Additionally, there were significant differences in preoperative ductions between the different surgical groups. More restriction in preoperative duction indicated greater fibrosis and a greater contractile force of the rectus, and this may be another reason for the different changes in strabismus and motility after decompression surgeries besides decompression extent.

Additionally, we found that a significant correlation existed between MR diameter and the increase in adduction and decrease in abduction. In previous studies, a strong trend toward increased motility restriction with increased muscle diameter was observed in TED patients [18]. MR was more frequently involved than LR in TED, which may be why esotropia increased more frequently after decompression. No significant difference was found between the change in vertical deviation and ocular duction, possibly due to the small sample and the limited extent of inferior wall decompression.

The main limitations of this study were the sample size and the relatively short follow-up, as many patients required bilateral decompression surgeries simultaneously or within a short period of time. The change in ocular deviation during the postoperative recovery period was demonstrated to be typically small [15].
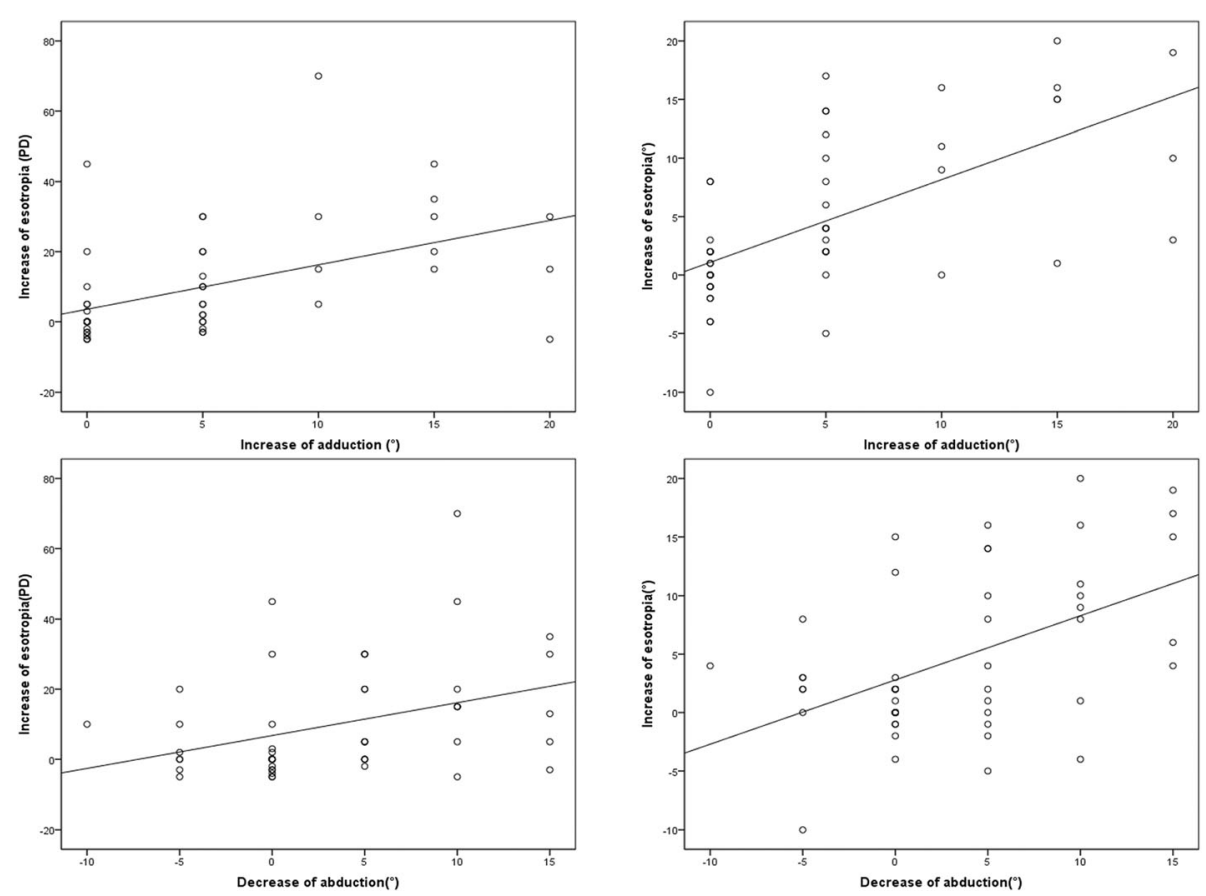

Fig. 3 The relationship between the changes in ocular ductions and deviations after orbital decompression. The increase in esotropia had a significant correlation with the increase in adduction and the decrease in abduction 

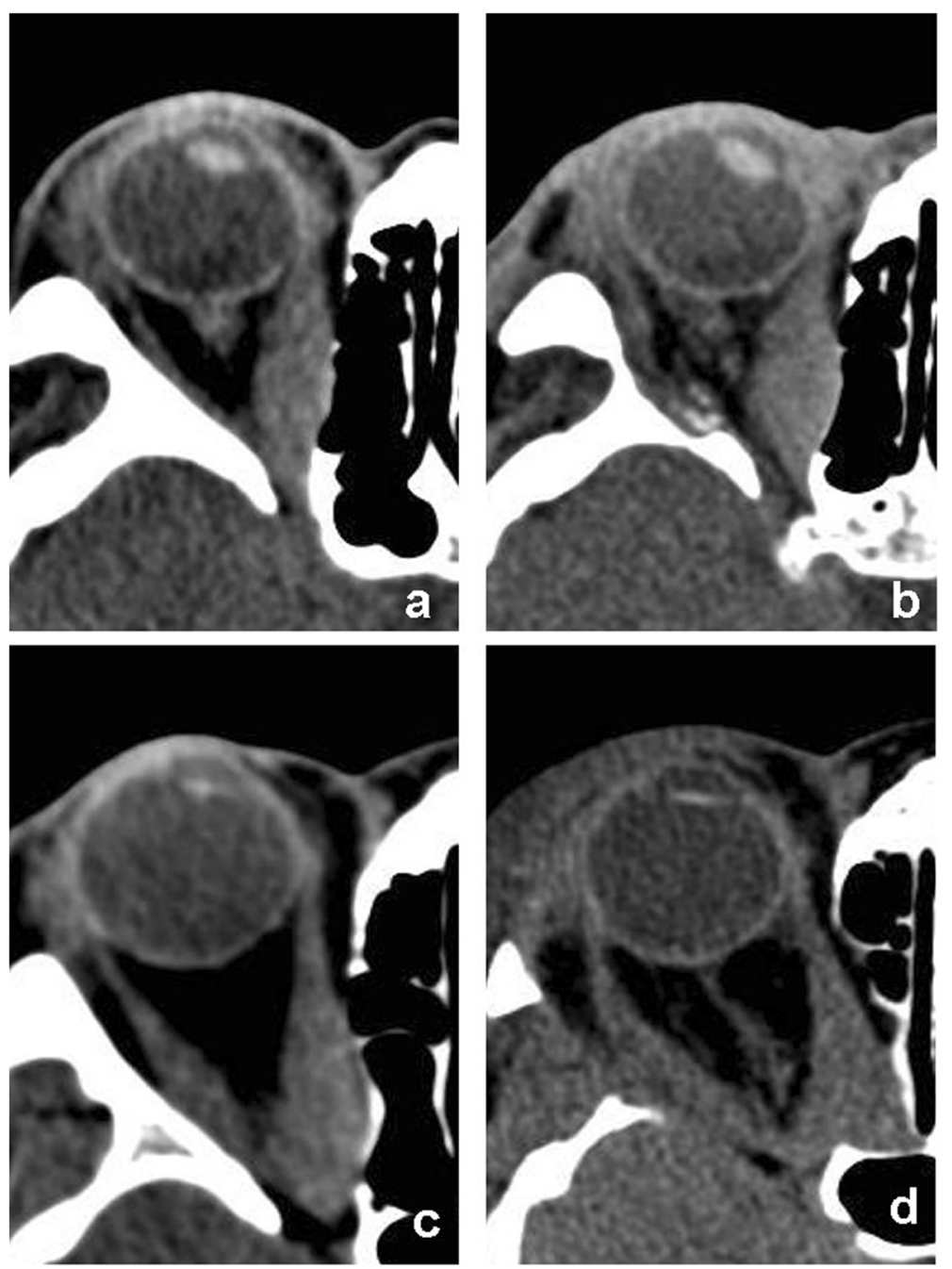

Fig. 4 Pre- and postoperative CT images of the patients who underwent orbital decompression. a-b A subject who underwent 1-wall decompression had an obvious increase in esotropia, the medial rectus became thicker after surgery. $\mathbf{c}$-d A subject who underwent 2-wall decompression had no worsening of strabismus. The position of the medial rectus changed but the thickness did not change much

In conclusion, the changes in ocular deviation and duction were different after 1-wall, 2-wall and 3-wall decompression. The increased contractile force of the rectus may be an important reason for the strabismus changes after orbital decompression.

\section{Abbreviations}

TED: Thyroid eye disease; NOD: New-onset diplopia; EOM: Extraocular muscles; CAS: Clinical activity score; CT: Computed tomography; MR: Medial rectus; LR: Lateral rectus

\section{Acknowledgements}

Not applicable.

\section{Authors' contributions}

Each author contributed to the conception and design of the work; J Guo and XF Li contributed to the acquisition and analysis of data; J Guo drafted the work; and RQ Ma and J Qian substantively revised it. The authors read and approved the final manuscript.

\section{Funding}

This study was supported by the National Natural Science Foundation of China [grant number 82000940, 81800867 and 81970835].

Availability of data and materials

All data generated or analysed during this study are included in this published article.

\section{Declarations}

Ethics approval and consent to participate

The study adhered to the tenets of the Declaration of Helsinki and was approved by the Ethics Committee of Fudan University. Informed consent was obtained from all patients in the study.

\section{Consent for publication}

Not applicable.

\section{Competing interests}

The authors declare that they have no competing interests. 


\section{Author details}

'Eye Institute and Department of Ophthalmology, Eye \& ENT Hospital, Fudan University, Shanghai, China. ${ }^{2} \mathrm{NHC}$ Key Laboratory of Myopia (Fudan University, Key Laboratory of Myopia, Chinese Academy of Medical Sciences, No. 83 Fenyang Road, Shanghai 200031, China. ${ }^{3}$ Laboratory of Myopia, Chinese Academy of Medical Sciences, No. 83 Fenyang Road, Shanghai 200031, China.

Received: 4 December 2020 Accepted: 23 February 2021

Published online: 12 March 2021

\section{References}

1. Borumandi F, Hammer B, Kamer L, von Arx G. How predictable is exophthalmos reduction in graves orbitopathy? A review of the literature. $\mathrm{Br}$ J Ophthalmol. 2011:95:1625-30.

2. Fichter N, Guthoff RF, Schittkowski MP. Orbital Decompression in Thyroid Eye Disease. ISRN Ophthalmology 2012;2012:10-5402.

3. Simon GJB, Syed AM, Lee $S$, et al. Strabismus after deep Lateral Wall orbital decompression in thyroid-related Orbitopathy patients using automated Hess screen. Ophthalmology. 2006;113:1050-5.

4. Alsuhaibani AH, Carter KD, Policeni B, Nerad JA. Orbital volume and eye position changes after balanced orbital decompression. Ophthal Plast Reconstr Surg. 2011;27:158-63.

5. Lee KH, Jang SY, Lee SY, Yoon JS. Graded decompression of orbital fat and wall in patients with Graves' Orbitopathy. Korean J Ophthalmol. 2014;28:1-11.

6. Kim KW, Byun JS, Lee JK. Surgical effects of various orbital decompression methods in thyroid-associated orbitopathy: computed tomography-based comparative analysis. J Craniomaxillofac Surg. 2014;42:1286-91.

7. Dolman PJ, Cahill K, Czyz CN, et al. Reliability of estimating Ductions in thyroid eye disease. OPHTHALMOLOGY. 2012;119:382-9.

8. Simon GJB, Wang L, McCann JD, Goldberg RA. Primary-gaze diplopia in patients with thyroid-related Orbitopathy undergoing deep lateral orbital decompression with Intraconal fat Debulking: a retrospective analysis of treatment outcome. Thyroid. 2004;14:379-83.

9. Goldberg RA, Perry JD, Hortaleza V, Tong JT. Strabismus after balanced medial plus Lateral Wall versus Lateral Wall only orbital decompression for Dysthyroid Orbitopathy. Ophthal Plast Recons. 2000;6:271-7.

10. Gordberg RA, Kim AJ, Kerivan KM. The lacrimal keyhole, orbital door jamb, and basin of the inferior orbital fissue. Arch Ophthalmol. 1998:116:1618-24

11. Golan S, Gupta A, Goldberg RA. Double vision after minimally invasive orbital decompression. J Craniofac Surg. 2017;28:e412-5.

12. Finn AP, Bleier B, Cestari DM, et al. A retrospective review of orbital decompression for thyroid Orbitopathy with endoscopic preservation of the Inferomedial orbital bone strut. Ophthal Plast Reconstr Surg. 2017:33:334-9.

13. Abramoff MD, Kalmann R, de Graaf MEL, Stilma JS, Mourits MP. Rectus Extraocular muscle paths and decompression surgery for graves Orbitopathy: mechanism of motility disturbances. Invest Ophthalmol Vis Sci. 2002;:43:300-7.

14. Sasim IV, De Graaf MEL, Berendschot TTJM, Kalmann R, van Isterdael C, Mourits MP. Coronal or swinging eyelid decompression for patients with disfiguring Proptosis in graves' Orbitopathy? OPHTHALMOLOGY. 2005;112: 1310-5.

15. Rootman DB, Golan S, Pavlovich P, Rootman J. Postoperative changes in strabismus, Ductions, Exophthalmometry, and eyelid retraction after orbital decompression for thyroid Orbitopathy. Ophthal Plast Reconstr Surg. 2017 33:289-93.

16. Tian S, Bolzani R, Benassi M, Lennerstrand G. Eye muscle force development in thyroid-associated ophthalmopathy in different stages of disease. Acta Ophthalmol. Scand 2007:85:431-7.

17. Tian S, Lennerstrand G, Nishida Y, Tallstedt L. Eye muscle force development and saccadic velocity in thyroid associated ophthalmopathy. Graefes Arch Clin Exp 2003;241:740-6.

18. Dagi LR, Zoumalan Cl, Konrad H, Trokel SL, Kazim M. Correlation between Extraocular muscle size and motility restriction in thyroid eye disease. Ophthal Plast Reconstr Surg. 2011;27:102-10.

\section{Publisher's Note}

Springer Nature remains neutral with regard to jurisdictional claims in published maps and institutional affiliations.

\section{Ready to submit your research? Choose BMC and benefit from}

- fast, convenient online submission

- thorough peer review by experienced researchers in your field

- rapid publication on acceptance

- support for research data, including large and complex data types

- gold Open Access which fosters wider collaboration and increased citations

- maximum visibility for your research: over $100 \mathrm{M}$ website views per year

At BMC, research is always in progress.

Learn more biomedcentral.com/submissions 